\title{
Conhecimentos e práticas de gerentes e profissionais da ESF na prevenção das violências com adolescentes
}

\author{
Know-how and techniques of Family Health Strategy managers \\ and professionals in the prevention of violence against adolescents
}

Moysés Francisco Vieira Netto (https://orcid.org/0000-0002-8035-9763) ${ }^{1}$

Suely Ferreira Deslandes (https://orcid.org/0000-0002-7062-3604) ${ }^{1}$

Saint Clair S. Gomes Júnior (https://orcid.org/0000-0002-1554-943X) ${ }^{1}$

${ }^{1}$ Instituto Fernandes

Figueira, Fiocruz. Av. Rui

Barbosa 716, Flamengo.

22250-020 Rio de Janeiro

RJ Brasil.

mfvnetto@hotmail.com

\begin{abstract}
The scope of this study was to identify empowering factors for the actions of the Family Health Strategy (FHS) in coping with violence among adolescents. The sample was random from 61 teams of the Family Health Strategy of three municipalities. Questionnaires tabulated by EpiINFO 7.0, with bivariate analyses were used. Chi -square, Fisher and the "classification tree" were then used to analyze the experiences in this theme and the following issues: bonding, time since training completed, qualification, knowledge of public policies among others. There is acknowledgement of adolescents' vulnerability to violence, however, only two teams have ongoing prevention experiences. The prevailing opinion is that such activities are not the responsibility of the FHS. The study identifies interdependent variables that increase the likelihood of preventing violence and the promoting of a culture of peace among adolescents.

Key words Family Health Strategy, Prevention of violence, Adolescent, Health promotion, Culture of peace
\end{abstract}

Resumo Este estudo objetivou identificar fatores potencializadores das ações da Estratégia Saúde da Família (ESF) no enfrentamento das violências contra adolescentes. A amostra foi aleatória de 61 equipes da ESF de três municípios. Utilizouse questionários tabulados pelo programa EpiINFO 7.0, com análises bivariadas: qui-quadrado, Fisher e "árvore de classificação", para análise das experiências nessa temática e das seguintes questões - vínculo, tempo de formado, capacitação, conhecimento de políticas públicas, entre outras. Há o reconhecimento da vulnerabilidade dos adolescentes às violências, porém apenas duas equipes possuem experiências de prevenção com continuidade. Predomina a opinião de que tais atividades não competem à ESF. O estudo identifica variáveis interdependentes que aumentam a probabilidade da prevenção das violências e a promoção de cultura de paz entre adolescentes.

Palavras-chave Estratégia Saúde da Família, Prevenção de violência, Adolescente, Promoção de saúde, Cultura de paz 


\section{Introdução}

A Estratégia Saúde da Família (ESF), criada em 1994, tem como principal desafio a reorganização da atenção básica à saúde no Brasil, e é reconhecida pelo Ministério da Saúde (MS) e por pesquisadores como um importante agente planejador e executor de ações com ênfase na promoção da saúde ${ }^{1-9}$. Segundo a Política Nacional de Promoção de Saúde, cabe às equipes de ESF o acompanhamento sistemático dos agravos e riscos à saúde ${ }^{4} \mathrm{e}$ o compromisso de trabalhar no enfrentamento e na prevenção das violências ${ }^{10}$.

Nesse aspecto, observa-se a ampliação das possibilidades e expectativas em relação às ações dos profissionais que compõem as equipes da ESF, graças ao incentivo e respaldo político que recebem, a trabalharem sob outra forma de organização da demanda, que se contrapõe ao paradigma clínico/assistencialista, e a lidarem com uma população adscrita, em uma jornada de trabalho integral (manhã e tarde).

Todavia, as equipes da ESF têm tido dificuldades de incorporar ações de prevenção às violências, especialmente com adolescentes. $\mathrm{O}$ fenômeno da violência produz grave impacto no setor de saúde, relacionado às doenças da população e ao sofrimento como barreira de acesso para serviços médicos ${ }^{11}$.

Muitos profissionais da ESF mostram-se resistentes por não considerarem que essas ações são pertinentes ao setor de saúde ${ }^{8}$. Há autores que assinalam que um dos desafios decorre da imagem estereotipada do adolescente, visto como problemático e difícil de lidar. A desarticulação entre as instituições e os setores que poderiam atuar juntos com a área da saúde na criação de redes de suporte e proteção ${ }^{12}$, o desconhecimento dos profissionais da ESF sobre o processo de notificação e encaminhamentos ${ }^{13}$, a ausência de treinamento, leituras e discussões nessa temática no ambiente de trabalho ${ }^{14}$ também constituem desafios que precisam ser tratados na agenda da ESF.

Após a criação da Secretaria da Juventude e do Conselho Nacional de Juventude em 2005, foram consolidadas em 2010 as "Diretrizes Nacionais para a Atenção Integral à Saúde de Adolescentes e Jovens na Promoção, Proteção e Recuperação da Saúde"15,16, de conteúdo enfático quanto à prevenção às violências e ao reconhecimento da ESF como uma das principais estratégias de efetivação das diretrizes ${ }^{11}$.

Como tem demonstrado a literatura, os adolescentes e jovens representam um dos grupos etários de maior risco e vulnerabilidade tanto para sofrer quanto para cometer violências ${ }^{17}$. Segundo a Organização Mundial de Saúde (OMS), a cada ano, cerca de 200 mil jovens são assassinados, e outros, em um número incalculável, envolvem-se em comportamentos de alto risco para sofrer ou praticar violências ${ }^{18}$. No Brasil, as agressões sofridas por esse segmento etário inflacionam as taxas de homicídios e outras modalidades de violências, posicionando o país em oitavo lugar entre os países mais violentos do mundo ${ }^{19}$.

No cenário nacional, a Região Metropolitana da Grande Vitória (RMGV) se destaca, posicionando o Espírito Santo (ES), em 2012, na segunda colocação no ranking nacional de homicídio juvenil entre os estados brasileiros ${ }^{19}$. Com aproximadamente $57 \%$ de toda a população urbana do $\mathrm{ES}^{20}$, essa região é destaque também internacional, ocupando, em 2014, o $16^{\circ}$ lugar entre os centros urbanos mais violentos do mundo ${ }^{21}$. A capital Vitória está em quinto lugar em mortes de adolescentes e jovens por arma de fogo ${ }^{22}$, e em primeiro no ranking brasileiro de homicídios envolvendo adolescentes do sexo feminino, com taxas de violência urbana que representavam o dobro da taxa nacional ${ }^{19}$.

Traçadas essas considerações, o objetivo deste artigo é identificar os fatores que potencializam as ações dos profissionais da ESF no enfrentamento das violências entre adolescentes. Os fatores investigados foram: conhecimentos de políticas públicas, tempo e tipo de vínculo de trabalho, formação e reconhecimento da vulnerabilidade dos adolescentes na adoção de práticas preventivas.

\section{Metodologia}

Trata-se de um estudo de delineamento transversal para explorar e descrever a experiência e o conjunto de ações das ESF na prevenção da violência e na promoção de uma cultura de paz entre adolescentes.

\section{Campo}

Os dados foram obtidos junto aos gerentes das unidades básicas de saúde (UBS) e dos profissionais das ESF nos municípios de Serra, Vila Velha e Vitória. Esses municípios compõem a RMGV e foram selecionados por: serem grandes aglomerados urbanos; estarem entre os $100 \mathrm{mu}$ nicípios mais violentos do Brasil em homicídio juvenil; e por aceitarem participar desta pesquisa. 
Foram consideradas elegíveis para o estudo todas as ESF dos municípios que apresentaram cadastro no Sistema de Informação de Atenção Básica (SIAB) em junho de 2015, totalizando 131 equipes. Essas equipes se situam em diferentes locais da RMGV, muitas delas de difícil acesso ou que demandam autorizações em diferentes níveis de gestão, o que tornaria o trabalho de campo demasiadamente longo e oneroso. Em função desse cenário, optou-se por trabalhar com uma amostra representativa, calculada considerando uma prevalência de $10 \%$ das equipes realizando alguma ação de prevenção, um nível de confiança de $95 \%$ e um erro de estimativa de $5 \%$.

Utilizou-se uma estimativa de no mínimo $10 \%$ de equipes da ESF realizando ações de prevenção de violências com adolescentes, com base em opinião consensual de especialistas, participantes tanto da banca de qualificação quanto da banca de defesa da tese de doutoramento do primeiro autor desta pesquisa. Infelizmente, não existem estimativas prévias com base em dados de literatura.

Esses parâmetros forneceram para o levantamento de dados um mínimo de 19 equipes para Serra, 14 para Vila Velha e 32 para Vitória. Tais equipes representam $70 \%$ de todas as equipes implantadas na RMGV.

\section{Método de obtenção dos dados}

A seleção das equipes participantes em cada um dos três municípios estudados ocorreu de forma aleatória, sem reposição a partir da listagem de equipes implantadas da ESF cadastradas no SIAB. Equipes de profissionais da ESF e gestores das UBS foram os participantes da pesquisa na coleta dos dados, por meio de aplicação de questionários.

$\mathrm{O}$ número de sujeitos participantes variou entre as UBS e os municípios por dois motivos: 1) variação já esperada quanto ao número de ACS prevista na diversidade do número de usuários adscritos em cada território, dada pela fle-

xibilização do MS, que indica uma média, e não o número absoluto na relação entre habitantes/ equipes da ESF; 2) opção dada aos profissionais das UBS selecionadas de participarem ou não da pesquisa.

Quando analisado o perfil dos profissionais da ESF participantes, identifica-se: média de idade de 37 anos; maior participação de mulheres (72\%); e média de quatro anos de tempo de trabalho na ESF.

Os dados foram obtidos a partir de dois questionários semiestruturados e elaborados com base em três publicações: Diretrizes Nacionais para a Atenção Integral à Saúde de Adolescentes do MS ${ }^{15}$; Prevención de La Violência: La evidencia (OPAS) ${ }^{23}$; Global Status Report on Violence Prevention $2014(\mathrm{OMS})^{24}$, que trataram recentemente a prevenção da violência envolvendo adolescentes, com proposições para a adoção de estratégias que devem compor a agenda de promoção de saúde da ESF. Com base nesses documentos, o Quadro 1 apresenta o conjunto de questões consideradas para esta pesquisa.

O levantamento dos dados ocorreu de outubro de 2015 a julho de 2016 e contou com o apoio de alunos de uma faculdade de odontologia do ES aprovados na disciplina de saúde coletiva e submetidos a treinamento para a coleta. Os dois questionários (um voltado para os gerentes e outro para as equipes da ESF) eram autoaplicáveis e foram entregues pessoalmente aos gerentes nas UBS, ficando acordada a data de devolução dos mesmos já preenchidos. O questionário dos gerentes foi respondido individualmente pelo gestor de cada UBS. O da equipe da ESF foi respondido por cada uma delas que participou, após reunião interna para discutir e produzir o consenso das respostas. Dessa forma, cada equipe respondeu a um questionário, de modo a expressar o coletivo das práticas do grupo.

O principal motivo das perdas de informações no campo foi a recusa de cinco equipes da ESF em participar, alegando falta de tempo, de recursos financeiros para participação em pesquisas, de apoio da gestão municipal e desmotivação.

\section{Método de registro e análise dos dados}

As informações foram digitadas e armazenadas em banco de dados elaborado no programa EpiINFO 7.0, a seguir foi realizada a conferência dos registros.

Os dados foram analisados de modo a descrever o perfil de gestores e profissionais da ESF 
Quadro 1. Questões dos questionários de gerentes e equipes da ESF.

\begin{tabular}{|c|c|c|}
\hline & Gerentes & Equipes \\
\hline $\begin{array}{l}\text { Questões } \\
\text { fechadas }\end{array}$ & $\begin{array}{l}\text { 1. Caracterização demográfica (idade, } \\
\text { sexo); } 2 \text {. Perfil profissional (tempo de } \\
\text { formado, tempo de gestão); 3. Vínculo de } \\
\text { trabalho; satisfação com as condições de } \\
\text { trabalho; 4. Formação: graduação, pós- } \\
\text { graduação, formação em saúde coletiva; } \\
\text { satisfação com sua formação em saúde da } \\
\text { família; 5. Conhecimento das principais } \\
\text { normativas (leis e políticas públicas) } \\
\text { de enfrentamento das violências e de } \\
\text { promoção da cultura de paz, bem como } \\
\text { as de atenção à saúde dos adolescentes }\end{array}$ & $\begin{array}{l}\text { 1. Tempo de implantação da equipe; } 2 \text {. Situação e } \\
\text { vínculo profissional de cada membro da equipe; } 3 \text {. } \\
\text { Perfil de formação dos membros da equipe (tempo } \\
\text { de formado, cursos técnicos e de pós-graduação); } 4 \text {. } \\
\text { Inclusão da temática da violência e sua prevenção na } \\
\text { formação recebida; participação, nos últimos anos, em } \\
\text { formações sobre prevenção da violência; caracterização } \\
\text { da formação recebida; } 5 \text {. Conhecimento das principais } \\
\text { normativas (leis e políticas públicas) de enfrentamento } \\
\text { das violências e de promoção da cultura de paz, } \\
\text { bem como as de atenção à saúde dos adolescentes; } 6 \text {. } \\
\text { Identificação de grupos etários mais vulneráveis às } \\
\text { violências no território; identificar se alguma ação } \\
\text { de prevenção é realizada com esses grupos etários; } 7 . \\
\text { Reconhecimento da equipe quanto ao papel das ESF } \\
\text { diante da prevenção de violências; } 8 \text {. Ações realizadas } \\
\text { com adolescentes do território }\end{array}$ \\
\hline $\begin{array}{l}\text { Questões } \\
\text { abertas }\end{array}$ & & $\begin{array}{l}\text { 1. Relato de situações de violências perpetradas contra } \\
\text { adolescentes no território da ESF. } \\
\text { 2. Relato das iniciativas de prevenção às violências e } \\
\text { promoção da cultura de paz junto aos adolescentes } \\
\text { realizadas pela equipe (relato de objetivos, frequência, } \\
\text { metodologia, resultados). }\end{array}$ \\
\hline
\end{tabular}

Fonte: Elaborado pelos autores.

e suas relações no desenvolvimento de atividades de prevenção às violências e de promoção de uma cultura de paz entre adolescentes.

Os resultados foram selecionados a partir das questões de maior evidência/correlação com as práticas dos profissionais da ESF com enfoque para ações específicas e consolidadas de prevenção à violência envolvendo adolescentes em seus territórios adscritos. Após a seleção, os resultados foram agrupados por município, levando em consideração o tamanho da amostra, o número de questionários respondidos, a fim de balizar os resultados para garantir a confiabilidade dos dados das inferências entre esses municípios.

Todas as UBS dos municípios estudados possuem semelhanças quanto ao processo de trabalho. De acordo com as entrevistas com os gestores das UBS, os municípios adotam minimamente os princípios e diretrizes preconizados pelo MS. Também foi consensual entre gestores e equipes da ESF que o modo de "fazer saúde" na ESF é uma discussão frequente nas secretarias municipais de saúde e na Secretaria Estadual do ES, com a realização de seminários, cursos de capacitação e até de especialização.
Em relação às características sanitárias, podese constatar uma proximidade de perfis entre os municípios. O próprio desenho geográfico, o fato de serem vizinhos e pertencentes a um grande aglomerado urbano (contíguo) que compreende a Região Metropolitana da Grande Vitória (a capital do estado) contribuem para tais semelhanças.

As violências urbanas são os maiores problemas sociais e de saúde pública nessa região, em que o tráfico de drogas e de armas são os maiores responsáveis pelo envolvimento de adolescentes, ora como os maiores agressores, ora como os mais agredidos, na grande maioria das vezes culminando em homicídios. Nesse cenário, o município de Serra foi considerado o segundo mais violento do Brasil ${ }^{21}$, contudo, em um ranking dos 100 municípios mais violentos, Vitória e Vila Velha também estão incluídos.

Foi considerado como desfecho principal deste trabalho a realização de ações de prevenção de violências. Para caracterização desse desfecho, analisaram-se as respostas positivas referentes às ações de prevenção às violências voltadas para os adolescentes e seus objetivos, metodologia, 
frequência/periodicidade e atuação da equipe de profissionais da ESF.

A partir do desfecho principal, realizou-se uma análise bivariada para identificação de fatores relacionados em termos estatísticos. Os testes de qui-quadrado e de Fisher foram utilizados para avaliar diferenças estatisticamente significativas. Todas as análises foram realizadas considerando um nível de significância de 5\%.

Um modelo de árvore de classificação foi empregado para identificar as variáveis utilizadas para descrever o perfil de gestores e equipes que, estão de alguma forma, relacionadas com as ações de prevenção às violências.

Esses modelos permitem uma representação gráfica da estrutura de interação das variáveis independentes com o desfecho analisado e têm como principais vantagens: a ausência de pressupostos para a variável dependente; a possibilidade de inclusão de um grande número de variáveis independentes categóricas e numéricas em sua escala original; a identificação de relações lineares e não lineares; e a facilidade de interpretação do resultado final. Como desvantagens, destacam-se a instabilidade do modelo, uma vez que problemas nos dados podem provocar grandes alterações no modelo final, e a inexistência de coeficientes que possibilitem avaliar o peso e a direção da associação de determinada variável considerada importante no processo de classificação, como acontece nos modelos de regressão ${ }^{25-33}$.

Os diferentes algoritmos de classificação montam a árvore escolhendo os atributos (categorias das variáveis independentes), que subdividem o "nó raiz" (categorias da variável dependente) em "nós folhas" (interrelações) de modo a aumentar a probabilidade ou a qualidade da ocorrência.

Para este trabalho, a árvore de classificação foi montada a partir do algoritmo CRT (classification and regression tree). Este algoritmo seleciona as variáveis independentes de forma a maximizar a homogeneidade dos atributos, melhorando assim a qualidade da classificação. O índice de Gini foi utilizado para avaliar a homogeneidade dos atributos a cada variável independente selecionada.

Para fins de simplificação e melhor visualização, optou-se por ocultar as ramificações da árvore referentes à decisão de não empreender ações de prevenção para as violências.

\section{Questões éticas}

Este estudo encontra-se registrado na plataforma Brasil e aprovado pelo Comitê de Ética e Pesquisa, conforme a Resolução 466/2012.

\section{Resultados}

\section{Perfil dos gerentes de UBS e profissionais da ESF e condições de trabalho}

O estudo envolveu 33 UBS (11 em Serra, 5 em Vila Velha e 18 em Vitória), 33 gerentes de UBS e 61 equipes de profissionais da ESF nesses municípios, totalizando 671 profissionais participantes.

Dos gerentes, $81,8 \%$ são do sexo feminino, na maioria com formação em enfermagem $(46,2 \%)$. Com relação ao tempo de formado, $86,6 \%$ concluíram a graduação depois da criação e implantação da ESF no Brasil (1994), e 72\% consideraram que o ensino que receberam sobre a ESF foi ótimo/bom.

A maior parte dos gerentes $(78,6 \%)$ tem pósgraduação em saúde coletiva, apenas 33,3\% têm vínculo efetivo e, para $87,8 \%$ deles, as condições de trabalho foram classificadas em ótimas ou boas. Entre esses, 29,7\% consideram que determinados problemas interferem na realização das atividades clínicas e de promoção, prevenção e educação em saúde, tais como: deficiência grave na infraestrutura da UBS; falta e troca constante de médicos; ausência de vínculo (profissionais/ usuários); microáreas descobertas por ACS e falta de computadores para gerar relatórios; burocratização no desenvolvimento das atividades extraclínicas; falta de organização do trabalho em equipe; dificuldade de relações interpessoais (entre os profissionais da equipe da ESF); falta de medicamentos; e baixa cobertura populacional pela ESF.

Nos municípios de Serra, Vila Velha e Vitória, respectivamente, $48,8 \%, 76 \%$ e $46 \%$ dos profissionais da ESF informaram possuir vínculo efetivo. Em Vitória, os enfermeiros e médicos apresentam altos percentuais de vínculo efetivo ( $96,7 \%$ e $58 \%)$, e em Vila Velha essas categorias apresentam percentuais bem mais baixos $(75 \% \mathrm{e}$ $25 \%)$. Em Serra, todos os enfermeiros são efetivos, mas os médicos $(27,7 \%)$ e ACS $(27,7 \%)$ possuem menor vínculo efetivo. Serra tem o maior percentual de profissionais que concluíram a graduação após a implantação da ESF (93,5\%), seguidos de Vila Velha $(73,8 \%)$ e Vitória $(64,3 \%)$. 


\section{Prevenção da violência e ESF: conhecimentos, valores e práticas}

Quando comparado o conhecimento entre gerentes e profissionais sobre as políticas e diretrizes públicas de cunho nacional vigentes que preconizam atividades de prevenção às violências com adolescentes e a promoção de uma cultura de paz, os gestores se destacam com maior grau de conhecimento (Gráfico 1).

Chama atenção que as normativas menos conhecidas por gerentes de UBS e equipes de ESF são justamente as especialmente destinadas ao enfrentamento das violências (Gráfico 1).

De acordo com os relatos dos profissionais das equipes da ESF sobre terem recebido algum tipo de formação em prevenção de violências de forma mais abrangente, ou com foco nos adolescentes, o município de Vila Velha destaca-se por ter o maior percentual de profissionais com formação complementar em prevenção às violências. Nos três municípios, o percentual de profissionais com formação em prevenção de violências com foco em adolescentes é maior do que o de profissionais com formação voltada para as violências na população em geral.

Entre os profissionais de nível superior da ESF, o CD obteve o menor percentual de formação em prevenção das violências para adolescentes $(28,3 \%)$. Os enfermeiros e ACS se declararam com maior formação, respectivamente $55,1 \%$ e $59,1 \%$.

Sobre a participação em capacitações, o município de Vila Velha foi o que mais capacitou seus profissionais $(54,6 \%)$. As secretarias municipais de Saúde, seguidas das secretarias estaduais, são as maiores responsáveis pela realização de capacitações. Em Vitória, Serra e Vila Velha, foram relatadas algumas parcerias e articulações com as equipes da ESF. Vitória foi o município com a maior descrição de articulações (35). São elas: Secretaria Estadual de Saúde, Segurança e Assistência Social; Centro de Referência Especializado de Assistência Social (CREAS); Proje-

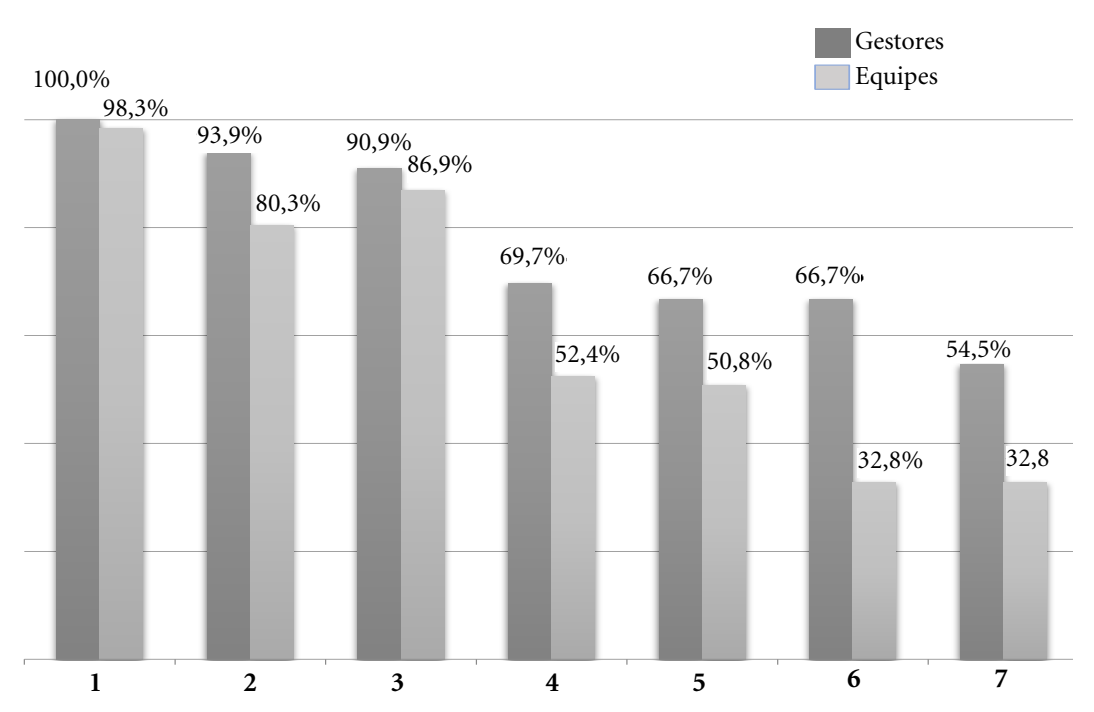

Políticas/normativas nacionais:

1. Estatuto da Criança e do Adolescente (ECA)

2. Política Nacional de Promoção de Saúde (PNPS)

3. Programa Saúde na Escola (PSE)

4. Diretriz Nacional de Atenção Integral à Saúde de Adolescentes (DNSA)

5. Linha de Cuidado - atenção integral à saúde de crianças e adolescentes e suas famílias em situação de violências (LC)

6. Por uma Cultura de Paz, a Promoção da Saúde e a Prevenção da Violência (CPPV)

7. Política Nacional de Redução da Mortalidade por Acidentes e Violências (PNRMAV)

Gráfico 1. Conhecimento dos gestores de UBS e equipes da ESF sobre as políticas públicas e outras normativas nacionais de proteção e prevenção às violências e promoção de uma cultura de paz envolvendo adolescentes. Serra, Vila Velha, Vitória - Espírito Santo, Brasil, 2016. 
to Caminhando Juntos (CAJUN); Médicos sem Fronteiras; e Ministério da Saúde. Em Serra, as 24 articulações descritas foram: 14 delas realizadas entre a própria Secretaria Municipal de Saúde, e dez com a Secretaria Estadual de Saúde. Em Vila Velha foram apenas três articulações: uma com a própria Secretaria Municipal de Saúde, uma com a Secretaria Estadual de Saúde e outra com a Secretaria Municipal de Saúde de Vitória.

O reconhecimento da vulnerabilidade e risco de adolescentes às violências foi assinalado em $77 \%$ das equipes. Essa percepção é mais acentuada em Serra (83,3\%). Em Vitória foi onde houve o maior número de descrição de manifestações de violências (Tabela 1).

A maioria das equipes $(78,7 \%)$ considera importante desenvolver atividades de prevenção às violências, contudo, apenas 19,7\% delas reconhecem essas atividades como de responsabilidade da ESF. Apesar desses dados, 20 equipes de Vitória $(64,5 \%)$ e dez de Serra $(55,6 \%)$ assinalaram desenvolver ou já ter desenvolvido ao menos alguma vez (sem determinação de intervalo temporal) atividade de prevenção destinadas a adolescentes. Dessas, apenas 11 de Vitória e seis de Serra descreveram as atividades que supostamente desenvolvem: ações pontuais na sala de espera e/ou orientações durante as consultas. Esses dados podem indicar que o percentual declarado sobre as "práticas" está inflacionado, e dessa forma somente 17 das 61 equipes pesquisadas $(27,8 \%)$ foram consideradas para análises posteriores (Tabela 1).

Quando analisadas as descrições acerca das atividades desenvolvidas pelas 17 equipes, constata-se que em apenas duas (de uma mesma UBS de Vitória) são programáticas, sistemáticas, com definição dos objetivos e/ou da metodologia e/ou da periodicidade das ações.

Poucas equipes descreveram os locais onde realizam as atividades de prevenção às violências com os adolescentes em sua área adscrita. As UBS foram os locais de maior eleição, citadas por quatro equipes de Vitória e três de Serra. Cinco citaram as escolas e apenas duas os domicílios.

A metodologia mais descrita pelas 17 equipes nos dois municípios foi a conversa durante as consultas na UBS. Os temas vinculados à promoção de uma cultura de paz mais abordados foram: sexualidade, DST/AIDS e planejamento familiar.

A Tabela 2 traz variáveis que foram consideradas importantes para a análise das equipes, tendo em vista o desfecho examinado: realizar ações de prevenção às violências. Verifica-se, entretanto, que nenhuma das variáveis consideradas apresentou diferenças estatisticamente significativas.

O modelo de árvore de classificação foi então utilizado no sentido de identificar o conjunto de variáveis que, articuladamente, contribuíram

Tabela 1. Valores e práticas sobre a prevenção das violências com adolescentes e a atuação das equipes de profissionais da ESF nos municípios de Serra, Vila Velha e Vitória, Espírito Santo, Brasil, 2016.

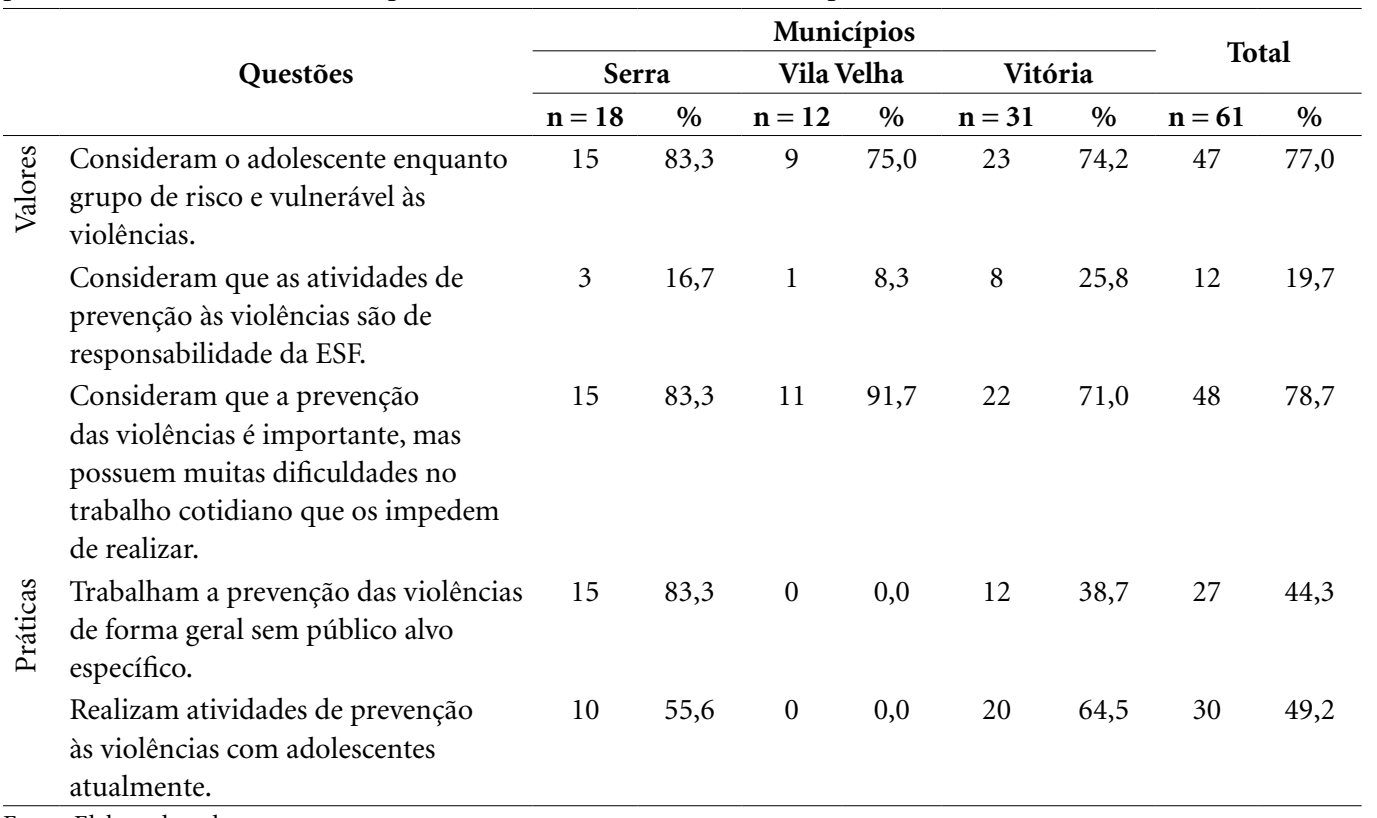

Fonte: Elaborado pelos autores. 
Tabela 2. Análise bivariada entre características, conhecimentos e atitudes das equipes da ESF de realizar ou não ações de prevenção às violências, a partir dos testes qui-quadrado e de Fisher.

\begin{tabular}{|c|c|c|c|c|c|c|}
\hline Questões & Itens & $\operatorname{Sim}(n=17)$ & $\%$ & Não $(n=44)$ & $\%$ & p-valor \\
\hline \multirow{8}{*}{ 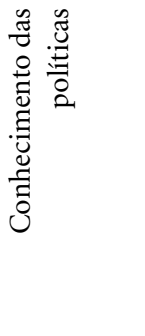 } & ECA & 17 & 100,0 & 43 & 97,7 & 0,721 \\
\hline & PNPS & 13 & 76,5 & 36 & 81,8 & 0,443 \\
\hline & PNRMAV & 6 & 35,3 & 14 & 31,8 & 0,511 \\
\hline & PSE & 16 & 94,1 & 37 & 84,1 & 0,281 \\
\hline & Diretriz & 11 & 64,7 & 21 & 47,7 & 0,183 \\
\hline & Orientação & 11 & 64,7 & 31 & 70,5 & 0,443 \\
\hline & Linha cuidado & 8 & 47,1 & 23 & 52,3 & 0,468 \\
\hline & Cultura paz & 5 & 29,4 & 15 & 34,1 & 0,489 \\
\hline \multirow{7}{*}{ 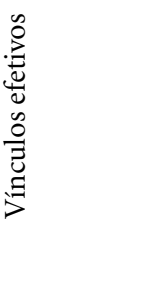 } & ACS & 11 & 64,7 & 29 & 65,9 & 0,402 \\
\hline & $\mathrm{CD}$ & 11 & 64,7 & 11 & 25,0 & 0,422 \\
\hline & ASB & 13 & 76,5 & 26 & 59,1 & 0,058 \\
\hline & TSB & 10 & 58,8 & 17 & 38,6 & 0,131 \\
\hline & ENF & 16 & 94,1 & 41 & 93,2 & 0.899 \\
\hline & T ENF & 10 & 58,8 & 30 & 68,2 & 0,442 \\
\hline & MED & 10 & 58,8 & 16 & 36,4 & 0,059 \\
\hline \multirow{7}{*}{ 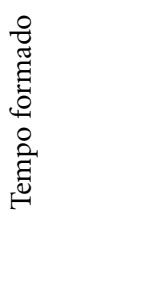 } & ACS & - & - & - & - & $\mathrm{n} / \mathrm{a}$ \\
\hline & $\mathrm{CD}$ & 0 & 0,0 & 1 & 2,3 & 0,422 \\
\hline & ASB & 1 & 5,9 & 3 & 6,8 & 0,667 \\
\hline & TSB & 1 & 5,9 & 1 & 2,3 & 0,667 \\
\hline & ENF & 2 & 11,8 & 13 & 29,5 & 0,181 \\
\hline & T ENF & 3 & 17,6 & 6 & 13,6 & 0,181 \\
\hline & MED & 4 & 23,5 & 10 & 22,7 & 0,400 \\
\hline \multirow{7}{*}{ 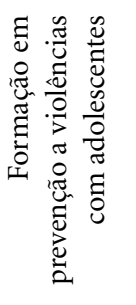 } & ACS & 12 & 70,6 & 20 & 45,5 & 0,126 \\
\hline & $\mathrm{CD}$ & 3 & 17,6 & 7 & 15,9 & 0,597 \\
\hline & ASB & 1 & 5,9 & 6 & 13,6 & 0,269 \\
\hline & TSB & 0 & 0,0 & 6 & 13,6 & 0,080 \\
\hline & ENF & 8 & 47,1 & 19 & 43,2 & 0,500 \\
\hline & T ENF & 5 & 58,8 & 14 & 31,8 & 0,388 \\
\hline & MED & 6 & 37,5 & 17 & 38,6 & 0,457 \\
\hline \multirow{6}{*}{ 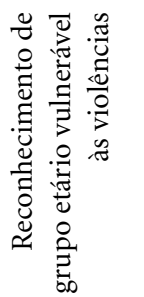 } & Criança & 13 & 76,5 & 34 & 77,3 & 0,597 \\
\hline & Jovens & 9 & 76,5 & 26 & 77,3 & 0,439 \\
\hline & Idoso & 13 & 76,5 & 31 & 77,3 & 0,448 \\
\hline & Adolescente & 15 & 76,5 & 33 & 77,3 & 0,221 \\
\hline & Adulto & 4 & 76,5 & 13 & 77,3 & 0,448 \\
\hline & Mulher & 13 & 76,5 & 28 & 77,3 & 0,260 \\
\hline
\end{tabular}

Fonte: Elaborado pelos autores.

para a probabilidade de ocorrência de ações de prevenção às violências.

Observa-se, na Figura 1, que as equipes que possuem médicos, ASB e TBS com vínculos efetivos, ACS com formação complementar em violência e conhecimento de quatro ou mais políticas/diretrizes nacionais com enfoque para essa temática aumentaram a probabilidade de ações de prevenção de $27,9 \%$ para $80,2 \%$. Entre os gestores, o conhecimento de quatro ou mais políti- cas/diretrizes nacionais com enfoque para a temática, acrescido de vínculo efetivo, aumentou a probabilidade de desenvolver ações de prevenção de violências de $42,4 \%$ para $72,7 \%$.

\section{Discussão}

A atuação das equipes da ESF estudadas frente às violências com adolescentes é motivo de preo- 

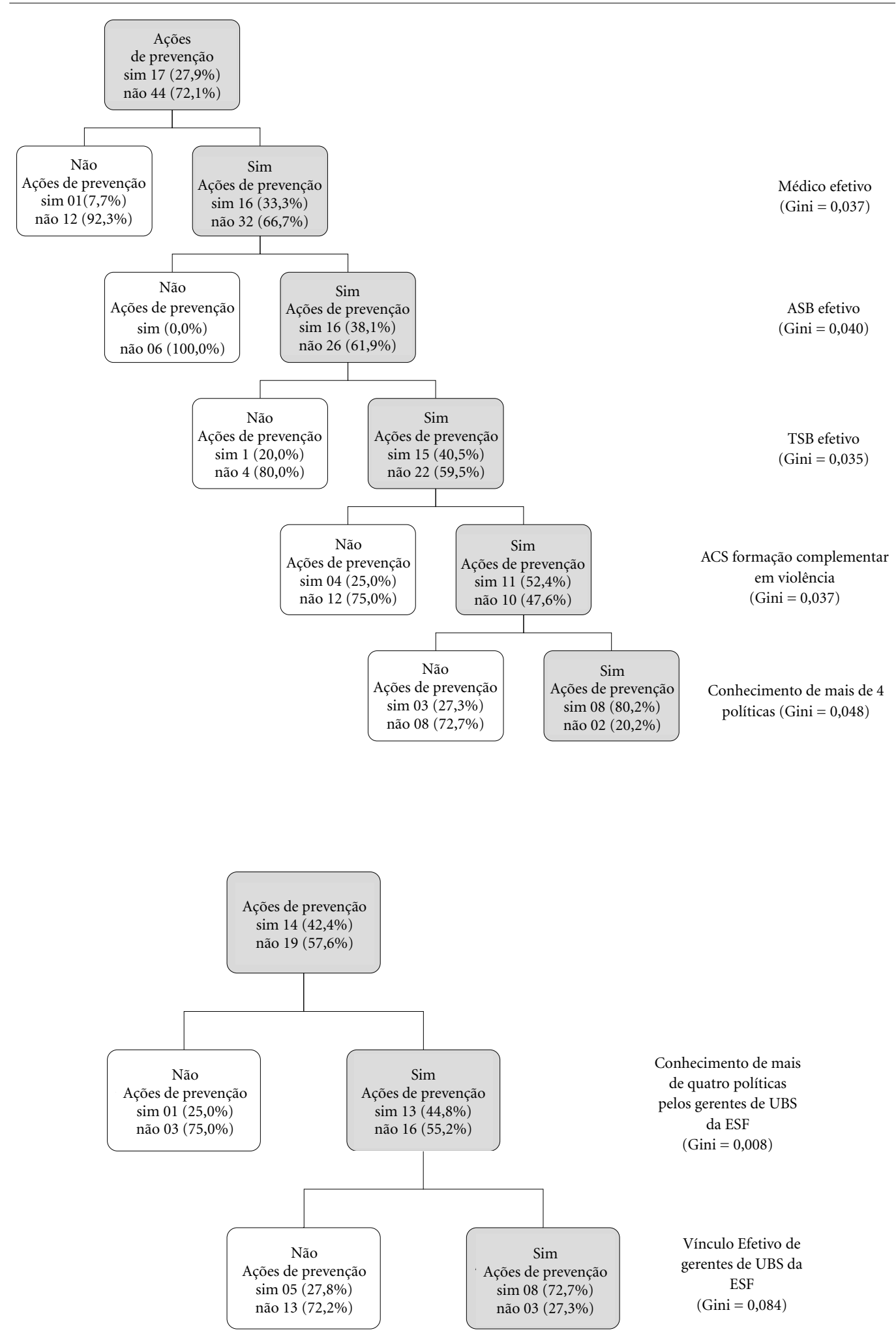

Figura 1. Modelo final de árvore de classificação ajustado para as equipes e gerentes de UBS da ESF dos municípios de Serra, Vila Velha e Vitória - Espírito Santo, Brasil, 2016.

Fonte: Elaborado pelos autores. 
cupação. Os gerentes das UBS e os profissionais descreveram atuações muito incipientes, esporádicas e restritas ao nível da rede de atendimento.

O presente estudo revelou um percentual elevado de gestores e profissionais da ESF que desconhecem as políticas que tratam da temática e de poucas equipes que receberam capacitação sobre prevenção de violências. A falta de preparo dos profissionais para atuar com os adolescentes é reconhecida pela literatura como um empecilho ${ }^{34}$.

A maioria dos pesquisados classificou como ótimo e bom o conteúdo que receberam acerca da ESF na graduação. Porém, confirmando estudo sobre o tema ${ }^{34}$, apenas pouco mais da metade dos enfermeiros e médicos descreveram ter recebido formação que lhes preparassem para desenvolver atividades de prevenção às violências.

Os ACS com melhor formação em prevenção às violências com adolescentes já trabalhavam a dinâmica do vínculo com a comunidade mesmo antes da implantação da ESF no Programa de Agentes Comunitários de Saúde (PACS), criado em 1991. Os estudos descrevem a proatividade desses profissionais, seja pela identificação pessoal com a proposta da ESF, seja pelo fato de partilharem da mesma comunidade, tendendo a uma maior identificação com os usuários ${ }^{35}$. Todavia, nem esses profissionais se destacaram na realização de ações de prevenção das violências.

Apesar do baixo percentual de profissionais da ESF que conhecem as normativas instituídas sobre a prevenção das violências com adolescentes e a promoção de uma cultura de paz, a grande maioria reconhece a vulnerabilidade dos adolescentes às violências. No entanto, essas percepções favoráveis não estiveram associadas às práticas efetivas de prevenção pelas equipes da ESF.

Chama atenção o fato de que 48 das 61 equipes de ESF pesquisadas reconhecem a necessidade de se trabalhar atividades de prevenção às violências com adolescentes, mas 49 delas não se consideram responsáveis por essas atividades. A baixa implicação das equipes pode ser um elemento relevante para entender por que menos de $1 / 3$ delas realizam atividades com tal foco. E dessas, somente duas equipes de uma mesma UBS as fazem de forma sistemática.

As dificuldades de compreensão e corresponsabilização das equipes da ESF na produção da promoção de saúde se mostram muito relevantes, pois ameaçam desqualificar a ESF enquanto referência para a reorientação da atenção primária à saúde, bem como de estratégia de reconhecido potencial para trabalhar as violências e a promoção de uma cultura de paz.
As manifestações de violências envolvendo adolescentes com mais citações pelas equipes, em ordem decrescente, são: o tráfico de drogas, a violência física, a violência sexual e os homicídios. Três equipes relataram ter medo da violência que o tráfico de drogas tem causado, pois já vivenciaram em suas UBS o "toque de recolher". Contudo, esses problemas não são tratados pelas equipes. Aquelas que descreveram atuar na prevenção das violências/promoção de uma cultura de paz com adolescentes desenvolvem sobretudo atividades relacionadas à sexualidade, às DST/AIDS e ao planejamento familiar. Observa-se um descompasso entre o que é diretamente identificado como risco e agravo à saúde dos adolescentes, especialmente acerca das formas de violências reconhecidas pelas equipes nos territórios da ESF e o que é executado nas UBS.

Os locais apontados pelas equipes da ESF como os de maior eleição para a realização das atividades foram os consultórios das UBS. Porém, os adolescentes são os usuários com menor frequência nas UBS ${ }^{36}$. Nesse sentido, pensar em uma rotina de prevenção às violências a esse grupo etário durante as consultas parece pouco abrangente e efetiva. Seria necessário levar em consideração essas peculiaridades dos adolescentes, estabelecendo estratégias de captação, ampliação do acesso, potencialização das relações e rompimento da barreira física da UBS, com a implementação de práticas coletivas, valorização dos ambientes de circulação e maior convívio dos adolescentes nos territórios.

Como visto no estudo, nenhuma das variáveis relativas às condições de trabalho, vínculo profissional, formação da ESF sobre o tema e o reconhecimento da vulnerabilidade e do risco dos adolescentes às violências contribuiu isoladamente para o desfecho examinado. Não foi possível discutir que tipo de formação foi recebida por esses profissionais, tanto que foi alto o entendimento equivocado de que a atuação face às violências não constitui prerrogativa do trabalho da ESF, a despeito do reconhecimento de que os adolescentes constituem grupo vulnerável a esse problema.

O reagrupamento das variáveis no modelo final de árvore de classificação, numa perspectiva multifatorial, identificou que há maior probabilidade de ocorrência de ações de prevenção de violências/promoção de uma cultura de paz quando os médicos possuem vínculo efetivo, o que sugere menor rotatividade desse profissional na ESF, possibilitando a criação de uma relação mais estável com a comunidade ${ }^{35}$. O mesmo foi 
constatado com o ASB e o TSB, sugerindo que o vínculo lhes proporciona maior oportunidade de trabalhar essa temática. De fato, esses profissionais (ASB e TSB) estão inseridos na ESF para otimizar o trabalho do CD em ações de educação e promoção de saúde, principalmente em ambientes escolares, com o aumento da cobertura de ações coletivas e maior efetividade na resposta às necessidades da população, segundo está previsto nas diretrizes ministeriais que oficializaram a inclusão do ASB e do TSB na ESF ${ }^{37}$. Pesquisadores analisaram a atenção à equipe de saúde bucal (ESB) na ESF e destacam as dificuldades dessa inserção $0^{9,38}$, não havendo registros na literatura de contribuições das ESB na ESF à prevenção das violências com adolescentes.

Constatou-se que o ACS tem maior probabilidade de contribuir com essa abordagem quando ele tem formação complementar e é conhecedor de quatro ou mais políticas/planos com enfoque nessa temática, indicando um nível de conhecimento mais aprofundado e um investimento feito em sua formação. Estudos já destacaram a contribuição do ACS para o entrosamento da equipe com a comunidade ${ }^{39,40}$. Essas pontes e a criação de vínculos têm contribuído para uma maior aproximação e intervenção de agravos na adolescência $^{40}$.

Nosso estudo mostra que o vínculo efetivo e o conhecimento das diretrizes por parte dos gerentes das UBS da ESF também aumentam a probabilidade de contribuição na prevenção das violências com adolescentes.

Há a necessidade premente de investimento na formação dos profissionais de saúde para atuação no enfrentamento à violência na atenção primária, principalmente na $\mathrm{ESF}^{41}$. Contudo, verifica-se que não basta ter o maior percentual de profissionais capacitados nessa temática e com vínculo efetivo, como demonstra o município de Vila Velha, que foi o município com o menor percentual de profissionais $(8,3 \%)$ que reconhecem que a prevenção das violências com adolescentes é também de responsabilidade da ESF e nenhuma de suas equipes descreveu a realização de atividades nesse âmbito.

Em Vitória, encontra-se a maior quantidade de profissionais que reconhecem o papel da ESF frente ao objeto desse estudo. Além disso, relatou-se menor dificuldade para desempenhar atividades de prevenção às violências. Entre as 61 equipes estudadas nos três municípios, em Vitória estão as duas únicas, de uma mesma UBS, que realizam um projeto contínuo e sistemático nessa temática. Por fim, observa-se que os gerentes e profissionais da ESF desse município demonstraram maior habilidade de articulação para obter parcerias e então criar as "redes de apoio, proteção e cuidado", algo imprescindível para a construção da intersetorialidade e a mobilização de outros atores sociais colaboradores ${ }^{14,42}$.

Face às constatações, cabe ressaltar que a interface violência no adolescer e a atuação da ESF necessita de maior empenho de estudiosos sobre o tema. Afinal, há consenso acerca da necessidade do direcionamento de novos estudos com aprofundamento de questões relativas à prevenção e ao reconhecimento pelo campo da saúde da família da violência envolvendo adolescentes, frente à predominância de vínculos ainda medicalocêntricos e à ausência de iniciativas de prevenção às violências, cerceadas pelas limitações do modelo biomédico e a ausência de tecnologias de enfrentamento e prevenção específicas ao problema apresentado ${ }^{43}$.

\section{Conclusão}

Conclui-se que - apesar do potencial da ESF previsto em suas diretrizes e por sua inclusão na agenda nacional de saúde do MS enquanto estratégia de reorganização da atenção primária à saúde no país, com vistas à redução dos agravos e a promoção da saúde e de uma cultura de paz - nos municípios estudados os gerentes e as equipes não promovem atividades sistemáticas de prevenção às violências com adolescentes, mesmo entre aqueles que vivem em territórios com percentuais altíssimos e crescentes das mais variadas manifestações de violências com adolescentes, incluindo aquelas que produzem vítimas fatais, medo e insegurança na comunidade, bem como transtornos nas atividades de trabalho das UBS.

Os resultados finais mostram a necessidade, por parte das secretarias municipais e estaduais de Saúde, de aumentar o investimento no conhecimento das diretrizes que tratam dessa temática, valorizar o vínculo efetivo dos profissionais e incentivar as parcerias junto a outros segmentos na implementação de atividades intersetoriais, com suporte de "redes de apoio e proteção". Contudo, nenhuma iniciativa dessas, quando analisadas isoladamente, exibe resultados significativos de proatividade das equipes da ESF nas ações de prevenção às violências com adolescentes.

O estudo aponta para a necessidade de implementações conjuntas, com variáveis interdependentes, em que uma iniciativa possa potencializar 
outra, e assim consecutivamente. Portanto, sugere-se a necessidade de investimentos diversos que apresentem questões como a discussão do papel das ESF frente às violências contra adolescentes, o vínculo efetivo de profissionais da ESF, a formação complementar, o conhecimento das po- líticas e diretrizes públicas na temática, além de gerentes de UBS com vínculo efetivo e conhecimento e capacitação em relação às políticas, diretrizes e normativas que tratam da prevenção das violências contra adolescentes, sob os princípios da promoção da saúde e de uma cultura de paz.

\section{Colaboradores}

Este artigo é produto do doutoramento de MFV Netto, que trabalhou na concepção e na redação final. De igual forma, SF Deslandes e SC Júnior contribuíram na concepção e na redação final do artigo. 


\section{Referências}

1. Brasil. Ministério da Saúde (MS). Programa de Saúde da Família. Brasília: MS; 1994.

2. Brasil. Ministério da Saúde (MS). Saúde da Família: uma estratégia para a reorientação do modelo assistencial. Brasília: MS;1998.

3. Brasil. Ministério da Saúde (MS). Secretaria de Atenção à Saúde. Departamento de Atenção Básica. Politica Nacional de Atenção Básica. Brasília: MS; 2006.

4. Brasil. Ministério da Saúde (MS). Política Nacional de Promoção da Saúde. 2a ed. Brasília: MS; 2007.

5. Santos AAG. Práticas e saberes de promoção da saúde para adolescentes na Estratégia Saúde da Família de Fortaleza [dissertação]. Fortaleza: Universidade de Fortaleza; 2011.

6. Primeiros passos do PSF. NoOlhar.com. [publicação na Internet]; 2004. [acessado 2005 Fev 20]. Disponível em: http://www.adm.noolhar.com/servlet/opovo?htm

7. Brasil. Ministério da Saúde (MS). Abrindo a porta para a dona saúde entrar [publicação na Internet]; 2005 [acessado 2005 Fev 19]. Disponível em: http:// www.saude.gov.br/psf//programas.

8. Goya N. Saúde da família: uma caminhada de 10 anos em prol do SUS. Revista CONASEMS 2004; 2(3):4445.

9. Almeida MCP, Mishima SM. O desafio do trabalho em equipe na atenção à Saúde da Família: construindo novas "autonomias" no trabalho. Interface - Comunic, Saúde, Educ; 2001;5(9):150-153.

10. Brasil. Ministério da Saúde (MS). Saúde e desenvolvimento da juventude brasileira: construindo uma agenda nacional. Brasília: MS; 1999.

11. Gonçalves HCB, Queiroz MRD, Godinho PG. Violência urbana e saúde mental: desafios de uma nova agenda? Fractal, Rev Psicol 2017; 29(1):17-23.

12. Njaine K, Assis SG, Gomes R, Minayo MCS. Redes de prevenção à violência: da utopia à ação. Cien Saude Colet 2007; 11(Supl.):1313-1322.

13. Zanelatto PF, Medeiros M, Santos WS, Munari DB. Violência contra crianças e adolescentes: significados e atitudes da Estratégia Saúde da Família. Rev Cienc Enfermagem 2012; 18(2):41-49.

14. Horta NC, Sena RR. Abordagem ao adolescente e ao jovem nas políticas públicas de saúde no Brasil: um estudo de revisão. Physis 2010; 20(2):475-495.

15. Brasil. Ministério da Saúde (MS). Secretaria de Atenção à Saúde. Departamento de Ações Programáticas e Estratégicas. Linha de Cuidado para a Atenção Integral à Saúde de Crianças, Adolescentes e suas Famílias em Situação de Violências: orientação para gestores e profissionais de saúde. Brasília: MS; 2010.

16. Pontes RP. Juventude(s) e políticas públicas. Rev Katálysis 2016; 19(1):9-10.

17. World Health Orzanization (WHO). Global status report on violence prevention. Geneva: WHO; 2014.

18. Waiselfisz JJ. Mapa da violência 2014: os jovens do Brasil. Rio de Janeiro: Flacso; 2014.

19. Instituto Brasileiro de Geografia e Estatística. Censo 2010 [publicação na Internet]; 2010. [acessado 2013 Out 31]. Disponível em: http://www.censo2010.ibge. gov.br/apps/mapa/
20. A Gazeta. Pesquisa mostra Grande Vitória como a 16a região mais violenta do mundo. A Gazeta dez 2013; p.1-2.

21. Waiselfisz JJ. Mapa da violência 2015: mortes matadas por armas de fogo. Brasília: Flacso; 2015.

22. Organização Panamericana de Saúde (OPAS). Prevención de la violencia: la evidencia. El Paso: OPAS; 2013.

23. Castelo GML, Venícios OLM, Gomes GN, Paixão $\mathrm{MAO}$, Farias O, Alves SN. Árvore de classificação para a avaliação do estilo de vida sedentário entre pessoas com hipertensão. Rev Investig Educ Enferm 2016; 34(1):113-119.

24. Chiavegatto ADP. Uso de big data em saúde no Brasil: perspectivas para um futuro próximo. Epidemiol Serv Saude 2015; 24(2):325-332.

25. Kajungu DK, Selemani M, Masanja I, Baraka A, Njozi M, Khatib R, Dodoo AN, Binka F, Macq J, D’Alessandro U, Speybroeck N. Using classification tree modelling to investigate drug prescription practices at health facilities in rural Tanzania. Malar J 2012; 11:311.

26. Kershaw TS, Lewis J, Westdahl C, Wang YF, Rising SS, Massey Z, Ickovics J. Using clinical classification trees to identify individuals at risk of STDs during pregnancy. Perspect Sex Reprod Health 2007; 39(3):141148.

27. Kuhn L, Page K, Ward J, Worrall-Carter L. The process and utility of classification and regression tree methodology in nursing research. J Adv Nurs 2014; 70(6):1276-1286.

28. Medeiros LB, Trigueiro DRSG, Silva DM, Nascimento JA, Monroe AA, Nogueira JA, Leadebal ODCP. Integração entre serviços de saúde no cuidado às pessoas vivendo com aids: uma abordagem utilizando árvore de decisão. Cien Saude Colet 2016; 21(2):543-552.

29. Song YLY. Decision tree methods: applications for classification and prediction. Shanghai Arch Psychiatry 2015; 27(2):130-135.

30. Souza A, Zaia JE. O uso do data mining na promoção de saúde: uma revisão sistemática da literatura. Atas de Saúde Ambiental 2015; 3(1):12-21.

31. Zhang H, Bonney G. Use of classification trees for association studies. Genet Epidemiol 2000; 19(4):323332.

32. Besen CBA. Estratégia Saúde da Família como objeto de educação em saúde. Rev Saude Soc 2007; 16(1):5768.

33. Kanno NP, Bellodi PL, Tess BH. Profissionais da Estratégia Saúde da Família diante de demandas médicosociais: dificuldades e estratégias de enfrentamento. Saude Soc 2012; 21(4):884-894.

34. Machado MFAS, Vieira NFC, Silva RM. Compreensão das mudanças comportamentais do usuário do Programa Saúde da família por meio da participação habilitadora. Cien Saude Colet 2010; 15(4):2133-2143.

35. Brasil. Ministério da Saúde (MS). Secretaria de Atenção à Saúde. Departamento de Atenção Básica. Saúde Bucal. Cadernos de Atenção Básica 17 (Série A. Normas e Manuais Técnicos). Brasília: MS; 2008.

36. Paim JS, Soares CLM. Aspectos críticos para a implementação da política de saúde bucal no município de Salvador, Bahia, Brasil. Cad Saude Publica 2011; 27(5):966-974. 
37. Cavalcanti MLT. Prevenção da violência doméstica na perspectiva dos profissionais de saúde da família. Cien Saude Colet 1999; 4(1):193-200.

38. Lima MCCS, Costa COM, Bigras M, Santana MAO, Alves TDB, Nascimento OC, Silva MR. Atuação profissional da atenção básica de saúde face à identificação e notificação da violência infanto-juvenil. Rev Baiana Saúde Pública 2011; 35(Supl. 1):118-137.

39. Moreira GAR, Vieira LJES, Deslandes SF, Pordeus MAJ, Gama IS, Brilhante AVM. Fatores associados à notificação de maus-tratos em crianças e adolescentes na atenção básica. Cien Saude Colet 2014; 19(10):4267-4276

40. Ramos MLCO, Silva AL. Estudo sobre a violência doméstica contra a criança em unidades básicas de saúde do município de São Paulo - Brasil. Saude Soc 2011; 20(1):136-146.

41. Machado JC, Rodrigues VP, Vilela ABA, Simões AV, Morais RLGL, Rocha EN. Violência intrafamiliar e as estratégias de atuação da equipe de Saúde da Família. Saude Soc 2014; 23(3):828-840.

42. Shimbo AY, Labronici LM, Mantovani MF. Reconhecimento da violência intrafamiliar contra idosos pela equipe da estratégia saúde da família. Esc Anna Nery 2011, 15(3):506-510.

43. Polaro SHI, Gonçalves LHT, Alvarez AM. Enfermeiras desafiando a violência no âmbito de atuação da Estratégia de Saúde da Família. Texto Contexto Enferm 2013, 22(4):935-942.

Artigo apresentado em 14/08/2018

Aprovado em 11/11/2019

Versão final apresentada em 13/11/2019

Editores-chefes: Romeu Gomes, Antônio Augusto Moura da Silva 